\title{
Meningitis and Reversible Cerebral Vasoconstriction Syndrome During the Postpartum Period
}

\author{
Mark I. Boulos, Joshua Wortsman, Richard H. Swartz, Peter C. Tai, Leanne K. Casaubon, \\ Jason Lazarou
}

doi:10.1017/cjn.2015.268

Can J Neurol Sci. 2015; 42: 341-343

Acute headache and focal neurological deficits should always prompt urgent investigation and management but in the postpartum period distinct diagnostic possibilities need to be entertained. A potentially important consideration is reversible cerebral vasoconstriction syndrome (RCVS), which typically has a favorable prognosis but can infrequently be fatal. Postpartum RCVS most commonly occurs after a normal pregnancy but a number of triggering factors have been identified; ${ }^{1}$ meningitis has not been previously reported as a precipitant.

One of the critical elements for a diagnosis of RCVS is a normal or near-normal cerebrospinal fluid (CSF) analysis, ${ }^{1}$ unless the situation is complicated by subarachnoid hemorrhage. In the patient described below, CSF changes consistent with an infectious process (but entirely negative infectious cultures) and the clinical context consistent with RCVS prompted consideration of aseptic meningitis as a trigger for her reversible vasculopathy.

\section{Clinical Presentation}

The patient was a 42-year-old female research consultant who was six weeks postpartum at the time of presentation to medical attention. She had undergone in-vitro fertilization with an uneventful pregnancy and delivery. Prior medical history included migraine with aura and postpartum hypothyroidism. Her current medications were naproxen, acetaminophen with codeine, and domperidone to aid with lactation.

Four weeks after her delivery (two weeks prior to hospital admission) she developed daily headaches which gradually increased in intensity over time. These headaches were qualitatively distinct from her migraine headaches in that they lacked a prodrome of nausea and her typical visual aura described as "TV lights or screen fuzziness". The patient took approximately two doses of sumatriptan during the first few days after the onset of the headaches; however, this medication did not provide relief and she discontinued its use. Her family doctor prescribed naproxen and acetaminophen with codeine for pain control.

On July 15, 2008, six weeks post-delivery, she experienced a severe thunderclap headache that evolved over the course of seconds and did not respond to naproxen or acetaminophen with codeine. Several hours after the onset of the thunderclap headache, she experienced aphasia and right-sided numbness, which developed over the span of fifteen minutes. She was transported to a local emergency room where her deficits progressed to include right-sided weakness. A code stroke was activated and examination by the on-call neurologist revealed right face and arm weakness, global aphasia, but no visual field deficit or abnormality with extra-ocular movements (National Institutes of Health Stroke Scale score of 10). Vital signs, screening blood work and computed tomography (CT) of her brain were normal. She was treated with intravenous tissue plasminogen activator for a suspected left middle cerebral artery stroke and transferred to the intensive care unit for monitoring.

The following day she became agitated and her headache worsened; her aphasia persisted but the limb weakness resolved. Examination revealed no meningeal signs, neck stiffness, rash or fever. Computed tomography angiography demonstrated diffuse narrowing of the proximal segments of the anterior and middle cerebral arteries, as well as of the terminal internal carotid arteries and right posterior cerebral artery (Figure 1A). Computed tomography of the brain, CT venogram and C-reactive protein were normal. Lumbar puncture revealed an elevated opening pressure $\left(42 \mathrm{~cm} \mathrm{H}_{2} \mathrm{O}\right)$, elevated white blood cell count $(1000$ $\mathrm{WBC} / \mathrm{mm}^{3} ; 59 \%$ lymphocytes, $41 \%$ neutrophils), high protein count $(1500 \mathrm{mg} / \mathrm{L})$, low glucose $(0.3 \mathrm{mmol} / \mathrm{L}$; serum glucose $6.8 \mathrm{mmol} / \mathrm{L}$ ), and normal red blood cell count. Infectious meningitis with secondary vasospasm was suspected and she was empirically treated with Ceftriaxone $2 \mathrm{~g}$ intravenously (IV), every 12 hours (q12h), Vancomycin 2 g IV q12h, Ampicillin 2 g IV q4h and Acyclovir $700 \mathrm{mg}$ IV q8h. Dexamethasone was considered but not administered because of the possibility of an active tuberculosis or Listeria infection.

Two days later (July 18, 2008) she made a remarkable clinical recovery with a normal neurological examination. Cerebrospinal fluid was tested for bacterial culture/gram stain, acid fast bacilli, amplified mycobacterium tuberculosis direct test, polymerase chain reaction for herpes simplex virus $1 \& 2$ and West Nile Virus, cryptococcal antigen test and fungal culture; all results were negative, and Acyclovir was discontinued. Cerebrospinal fluid cytology demonstrated a reactive lymphocytosis. Hypercoagulable and rheumatological screens were normal. She was discharged home three days later on no medications and with no recurrent focal neurological deficits. Prior to discharge, she had

From the Division of Neurology (MIB,JW, RHS), Department of Medicine, Sunnybrook Health Sciences Centre; Division of Neurology (PT,LKC,JL), Department of Medicine, University Health Network, Toronto, Ontario, Canada.

Received August 16, 2014. Final Revisions Submitted March 26, 2015.

Correspondence to: Mark I. Boulos, Sunnybrook Health Sciences Centre, Room A442 2075 Bayview Avenue, Toronto, Ontario, M4N 3M5, Canada. E-mail address: mark. boulos@sunnybrook.ca 

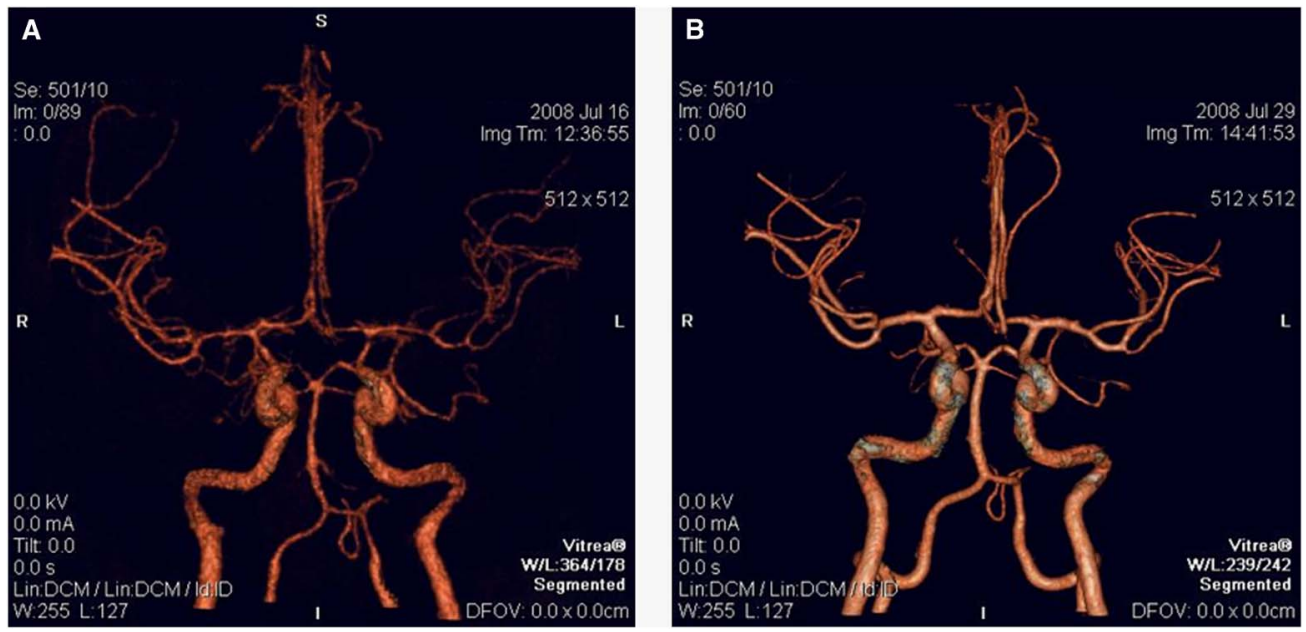

Figure 1: A. Initial CT angiogram demonstrated diffuse narrowing of the proximal segments of the anterior and middle cerebral arteries, as well of the terminal internal carotid arteries and right posterior cerebral artery. B. Two weeks after symptom onset, CT angiography demonstrated resolution of the original diffuse vasospasm.

been treated for five days with antibiotics but resolution of her symptoms had occurred 48 hours after initiation of therapy. Given the rapid resolution of her focal neurological deficits, magnetic resonance imaging (MRI) of the brain was not conducted during her hospital stay.

Two weeks after her original presentation to hospital repeat CT angiography was normal, with no evidence of vasospasm or vasculitis (Figure 1B). Mild headaches persisted after her discharge but resolved upon administration of prednisone as pre-medication for the CT angiogram; the patient had had an allergic reaction to CT contrast dye during an earlier study. Subsequent MRI and MR angiography performed two months post-discharge were also normal.

She has now been followed for six years after her event and no evidence of any new pathology has arisen. She occasionally experiences migraine headaches but is avoiding the use of triptan medications.

\section{DisCuSSION}

Our case demonstrates a postpartum female who presented with a thunderclap headache and symptoms suggestive of a left hemispheric stroke; her symptoms resolved within 72 hours of their onset. Initial CT angiography showed diffuse vasospasm, which resolved within two weeks. Cerebrospinal fluid demonstrated an elevated opening pressure and white blood cell count, high protein, and low glucose suggestive of an infectious process; however, all CSF cultures were negative, despite initiation of antibacterial and antiviral therapy after the lumbar puncture.

Aside from the abnormal CSF profile, the patient's condition was most in keeping with RCVS, for which the postpartum state is a common trigger. ${ }^{1}$ In this case, sumatriptan use ${ }^{1}$ (although minimal) and the prior history of migraines ${ }^{1}$ may have also served as additional precipitating factors.

The abnormal CSF profile described here is not in keeping with a diagnosis of isolated RCVS because such a diagnosis requires a normal or near-normal CSF analysis. ${ }^{1}$ In addition, no case series of patients with RCVS have identified a patient with a comparable CSF profile. Ducros et al. ${ }^{1}$ reported on 67 patients with RCVS, of which only $26 \%(16 / 62)$ had elevated CSF WBC counts, the highest being $35 \mathrm{WBC} / \mathrm{mm}^{3}$; in addition, $39 \%(24 / 62)$ had slightly elevated CSF protein levels, with a mean protein level of $59 \mathrm{mg} / \mathrm{L}$. Singhal et al. ${ }^{2}$ reviewed 139 RCVS cases: In patients who underwent CSF examination, $78 \%$ had normal findings and the remainder had minor abnormalities attributed to underlying stroke or other co-existing diseases; only $3 \%$ of their patients had CSF examinations demonstrating greater than $10 \mathrm{WBC} / \mathrm{mm}^{3}$, but the value of the highest WBC was not reported. In comparison, our findings of an elevated white blood cell count of $1000 \mathrm{WBC} / \mathrm{mm}^{3}$ and elevated protein count of $1500 \mathrm{mg} /$ $\mathrm{L}$ were distinctive. Furthermore, our patient had an elevated opening pressure and low glucose level, all of which favour an infectious process.

Despite the abnormal CSF profile indicative of a CNS infection, other clinical features suggestive of an infection were missing in our patient, such as a positive CSF culture, fever, rash or meningismus; in addition, there was prompt resolution of her symptoms within less than 72 hours of their onset. Because bacterial or viral meningitis could not be initially definitively ruled out, empirical treatment with antibiotics and antiviral medication was started after the lumbar puncture until culture results became available.

Given the absence of positive infectious cultures, our patient was given a presumed diagnosis of aseptic meningitis. Her use of naproxen may have served as a trigger for aseptic meningitis. We presume that her initial headaches, which occurred approximately two weeks prior to presentation, were the first manifestation of aseptic meningitis; spontaneous resolution took place four weeks after the first headache, consistent with a typical course for aseptic meningitis. While we did not successfully culture a viral cause, this is not uncommon - many cases of meningitis have no specific viral underlying etiology identified despite extensive work-up. ${ }^{3}$ In the realm of viral meningitides, varicella zoster virus (VZV) is the most typically associated with vasoconstriction, ${ }^{4}$ however, the vasculopathy seen in our patient was more diffuse than that which is typically associated with VZV. 
Aside from aseptic meningitis precipitating RCVS, another diagnostic consideration is the syndrome of transient "headache and neurologic deficits with cerebrospinal fluid lymphocytosis" (HaNDL) as a triggering factor for her RCVS. In a series of 50 patients with HaNDL, ${ }^{5}$ the highest reported CSF WBC was $760 \mathrm{WBC} / \mathrm{mm}^{3}$, greatest CSF opening pressure was $37 \mathrm{~cm} \mathrm{H}_{2} \mathrm{O}$, and highest protein level was $2500 \mathrm{mg} / \mathrm{L}$; all patients were noted to have normal CSF glucose levels. Our patient demonstrated slightly greater values for the CSF opening pressure and WBC values, and her CSF glucose was reduced. In addition, our patient's vascular imaging demonstrated diffuse vasospasm in contrast to the focal irregularities described in one patient of the same study ${ }^{5}$ and, subsequently, in another two patients. ${ }^{6}$ Therefore, her presentation is not typical for HaNDL and is more classic for aseptic meningitis.

With regards to other diagnostic possibilities, serial CT brain scans conducted on two consecutive days (upon arrival in the emergency room as a code stroke and on the following day) did not show any evidence of evolving brain infarction and follow-up MRI did not show a lesion consistent with a brain infarction; in addition, clinically she did not have a persistent focal neurological deficit suggestive of a stroke. Furthermore, imaging did not suggest Posterior Reversible Encephalopathy Syndrome (PRES); her normal blood pressure and the absence of seizures or visual disturbances also argued against PRES. Nonetheless, since an MRI was not conducted during the acute phase of her illness, the possibility of PRES cannot be definitively ruled out.

Treatment with calcium channel blockers or short-term high dose glucocorticoids has been suggested in patients with RCVS, particularly in those with recurrent headaches, severe vasospasm, or transient neurological symptoms. Our patient's benign course did not necessitate the use of further medications. Interestingly, our patient's mild residual headaches did respond to prednisone that was administered prior to a repeat CT angiogram for allergy prophylaxis.

Overall, we believe the patient's severe postpartum headaches were caused by meningitis of unclear etiology (presumed to be aseptic meningitis) which triggered RCVS. Her stroke-like symptoms may have been caused by hemodynamic changes occurring in the setting of RCVS. Although aseptic meningitis is relatively common, we suspect the association with RCVS had not been previously reported because of the underuse of neurovascular imaging in patients with typical presentations of aseptic meningitis. However, the more recent routine use of advanced neurovascular imaging permitted identification of the association with RCVS, as well as a link with reversible cerebral vasoconstriction (in the absence of $\operatorname{RCVS}^{7}$ ).

\section{CONCLUSION}

There is a broad and often challenging differential diagnosis that must be considered when presented with a postpartum patient experiencing neurological symptoms. The diagnosis of RCVS needs to be considered in patients with a severe headache and narrowing of the intracranial vasculature, especially when other causes have been ruled out and the clinical deficits and vascular narrowing are shown to resolve. ${ }^{1,2}$ Reversible cerebral vasoconstriction syndrome may be present in conjunction with a large number of other syndromes. Our case suggests that RCVS may be triggered by aseptic meningitis.

\section{ACKNOWLEDGEMENTS}

During the preparation of this manuscript, Dr. Boulos was supported by a Focus on Stroke Research Fellowship, which was funded by the Heart and Stroke Foundation of Canada, the Canadian Stroke Network and the Canadian Institutes of Health Research.

\section{Disclosures}

Mark Boulos has the following disclosures: Heart and Stroke Foundation, Researcher, Research support; CIHR, Researcher, Research support; Canadian Stroke Network, Researcher, Research support. Joshua Wortsman has nothing to disclose. Richard Swartz has the following disclosures: Heart and Stroke Foundation, Researcher, Research support; CIHR, Researcher, Research support; Ontario Brain Institute, Researcher, Research support. Peter Tai has the following disclosures: Inventive Health (CRO for Upsher-Smith), Clinical Trial Site PI, Research support. Leanne Casaubon has the following disclosures: Medtronic, Independent Neurologic Assessor, Consulting Fees; Covidien, Advisory Board Member, Honoraria; Bayer, Speaker, Speaking Honoraria; Heart and Stroke Foundation, Researcher, Grant; CIHR, Researcher, Grant; Ontario Brain Institute, Researcher, Grant; Brain Canada, Researcher, Grant. Jason Lazarou has nothing to disclose.

\section{REFERENCES}

1. Ducros A, Boukobza M, Porcher R, Sarov M, Valade D, Bousser MG. The clinical and radiological spectrum of reversible cerebral vasoconstriction syndrome. A prospective series of 67 patients. Brain. 2007;130:3091-101.

2. Singhal AB, Hajj-Ali RA, Topcuoglu MA, et al. Reversible cerebral vasoconstriction syndromes: Analysis of 139 cases. Arch Neurol. 2011;68:1005-12.

3. Kupila L, Vuorinen T, Vainionpaa R, Hukkanen V, Marttila RJ, Kotilainen P. Etiology of aseptic meningitis and encephalitis in an adult population. Neurology. 2006;66:75-80.

4. Chow FC, Marra CM, Cho TA. Cerebrovascular disease in central nervous system infections. Semin Neurol. 2011;31:286-306.

5. Gomez-Aranda F, Canadillas F, Marti-Masso JF, et al. Pseudomigraine with temporary neurological symptoms and lymphocytic pleocytosis. A report of 50 cases. Brain. 1997;120(Pt 7):1105-13.

6. Kappler J, Mohr S, Steinmetz H. Cerebral vasomotor changes in the transient syndrome of headache with neurologic deficits and csf lymphocytosis (handl). Headache. 1997;37:516-8.

7. Leung LY, Brett M, Zurawski J, Kumar S. Recurrent aseptic meningitis with reversible cerebral vasoconstriction. J Neurol Sci. 2014;339:239-40. 